\title{
UNIDADES DE TERAPIA INTENSIVA: CONSIDERAÇÕES DA LITERATURA ACERCA DAS DIFICULDADES E ESTRATÉGIAS PARA SUA HUMANIZAÇÃO
}

Intensive Care Units: considerations of literature about the difficulties and strategies for its humanization

Unidades de Terapia Intensiva: consideraciones de la literatura acerca de las dificultades y estrategias para su humanización

Fabiana Bolda

Marli deCarvalhoJericó

\section{Resumo}

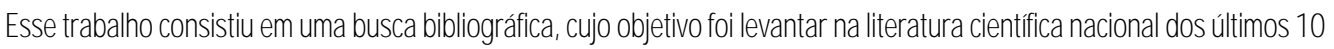

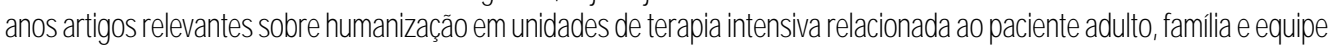

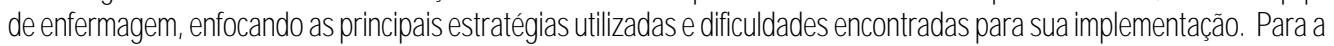
seleção dosartigos, foramestabdecidos critérios deindusão, e após a definição desses artigos, foi realizadale liturae análise descritiva dos mesnos. Os resultadbs obtidos coma análise dos dadbs identificaramque, apesar do tema "humanização" ter sido bastanteabordado nos útimos 10 anos, tal abordagemsedeudemodba conceituar, buscar

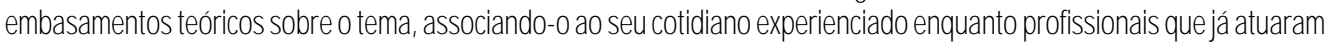

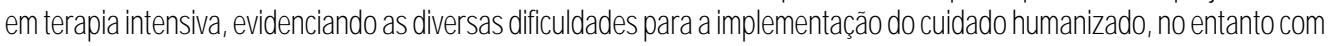
parassugestões vaáveis deestratégias parata.

Palaras dave Huraristo. Utidades de Terapialntensiva Erfermagem
Abstract
Resumen

This nork consists of a bibliographical search whose Esetrabajoconsistióen unabuscabibliográica ayodbiej-

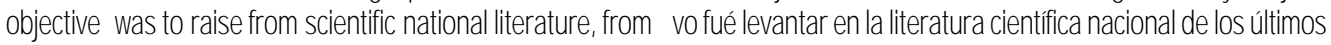
thelast 10 years, relevart artides about humarization in 10 añosatíalos relevertessobreh unarizacónen uridades intensivecare units related to theadit petient, familyand deterapiaintensivarelacionadaal pacienteadlto, faniliay nursingstaff foasing on theprinipal strdegies usedand equipedeerfermeria enfocandblas prinapales estrdegias thedffialtiesfoundforitsimplemettion Forthesedection utilizadas yodfialtades encortradas perasuimplenertación of theartides some indusioncriteriahavebeenstadished Paralaseleccióndelosartía losfueronestadecidosciteriosde andafter thedefinition of theseatides, ithas beennæadea indusión y después dela definioón deeses artíalos fueron reading and a describing analisys of them The results realizadas lectura yarálisis descriptivo de los nismos. Los dbtained with theanalisys of theinformationidentifiedthat, resultadbs dateridos conel análisisdelos catos identificaron

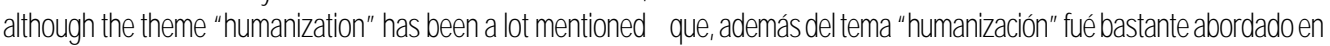

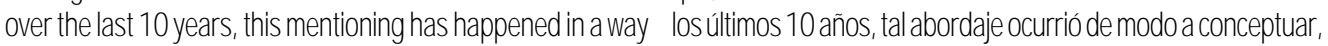

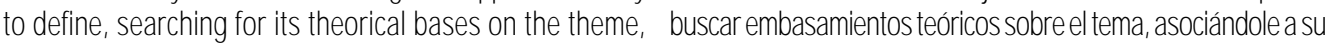
associatingits daylyexperiences whileprofessionals that cotidano vividben arato profesiondes queya acturenen have actually worked in intensive care units, showing the terapiaintensiva, evidenciandbas diversas dfialtades parala many diffialties for theimplementation of humarized care, implementación del aidado humarizad, pero con pocas

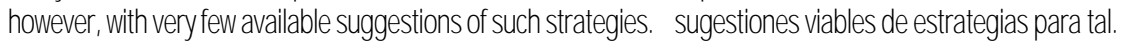

\author{
Keyords \\ Humanism IntensiveCare Lhits. Nursing.
}

\author{
Pabasdae \\ HstoriadelaEvermería Enser̃anza. Investigadón
}


INTRODUÇÃO

A formação médica e dos demais profissionais de saúde temprionizado a espedialização dos resmos e, comisso, o melhor preparo dos profissionais, cada vez mais capacitados e habilitados a oferecer o me Ihor tratanerto e conseqüente reaperação au ara do padiente Nb entanto, ta formação temdeixadb a singula ricade do padiente (seus valores, crencas, sentimentos e enroções) emsegundo plano, emdetinento do "fígado dbente", da "finção renal prejudicada", etc, o que tem comprometido significativamente 0 atendimento humarizadb e de boa qualidade nes instituições de saúde

Vsandb a una modança desse pandrana neaiond, o Mristéio da Saúde cria, em2001, o Programa Nacional de Hurrização da Assistência bspitdar (PNHAH) ${ }^{1}$, como dje tio de hunanzar a assistênia hospitdar prestada aos pacien tes denddos nos hospitais públicos, e, em2003, juntamente comos derais programas de humarizacão preevistertes, 0 PNHAH transforma-se em una Política Nacional de Hurrizaça, o H narizaSSB, abrangendb a saúde pública cono umtodb, ratertativaden methrar a qualidadee eficáda dos serviços prestadbs pelas instituçães de saúde

É nesse conteto que tantbémse faz necessário effocar a hunarização nes Unidades de Terapia Intensiva (UIIS). ParaSara et $a^{3: 7}$,

As Uticades de Terapia Intensiva (UTls) são conside radas como locais destinados à prestação de assistênda espedializada a padientes emestado cútico. Para os padientes á internados há necessidade de controle rigoroso dos seus parâm tros vitais e assis têndia de enfermagemcontína e intensiva.

Algumas caractenísticas peculiares de uma UT são: o ambiente permeado por tecnologia de ponta, situações iminentes de emergência e necessidade constante de agilidade e habilidade no atendimento ao diente

Apesar de toda a tecnologia empregada nas UTls e, comisso, o melhoramento da assistência prestada, o índice de mortalidade ainda é elevado, o que criou 0 mito, para pacientes e familiares, de que a UTI está diretamente relacionada à morte e a pacientes que não têmchance de reauperação(Souza đt al.3).

Na chulidade, vêse umpanorama nas Ulls que nos condz para a urgênaia da preoa pação como resgate da humarização. Há una naiơ preocupação como aperfeiçoamento da técrica, valonizandb o modb-de-sertrabalho, emdetrimento do modb-deser-aidadb(Boff') . Nestesentido, Corrêê:161 dz que:

Emmeio a aparelhagens e técricas complexas, é prediso buscar o humano que ali se encontra, não apenas enquanto aquele padiente que necessita ser constantemente monitorado emsuas funçoes vitais, mas comb o ser-á que vivendia a fadicidade da doença que o envolve emsua totalidade existendal e, semdúmida, o faz experiendiar a insegut rança do "poder ser saudável", enfrentando a doença e o risco da morte.

O conceito de humanização pode ser traduzido como una busca incessante do conforto físico e psíquico e espinitual do paciente, famlíla e equipe $\mathrm{No}$ contexto hospitalar, Mezomó, ao discutir o que é a humarização do hospital, entende que:

Humanizar significa agir sobre a sua administração e o seu funcionamerto, bemcomo a ditude do pessoal face ao enfermo, como djeivo de proporcionar-Ihe umanbiente físico e social tão agradável quanto possível, ressaltando os dissabores inevitáveis de seu tratanento.

Villa e Rossi ${ }^{7: 138}$ entendemque humanizar é uma medida que visa, soloretudo, tomar fetiva a assistênda ao indivíduo citicamente dbente, considerando-o como umser biopsicossocioespinitual.

A humarização emTerapia Intensiva surge na tentativa de melhorar a assistência prestada a pacientes cúticos e seus familiares e, também as condições de trabalho da equipe multidisciplinar atuante em Cl. Para Santos et al. 8:28:

A equipe miltiprofissional que tua nas UIIs é com posta por: Médicos Intensivistas, responsáveis pela assistêndia rédica durante a permanêndia do par derte na UII, que, juntamente como médico responsável pela internação do paciente, elabora um plano para diagnóstico e tratamento; Enfermeiras são responsáveis pela avaliação e elaboração de um plano de aidados de enfermagemindividualizadb e sistematizado. A equipe miltidisaiplinar da UTl ainda é composta por Auxiliar de Enfermagem Agente de Transporte, Auxiliar Administrativo, Aut xiliar de Higiene Hospitalar, Fisioterapeutas, Nutricionistas e Voluntánias.

É importante ressaltar que essa equipe multidisciplinar está unida por umobjetivo comm e que, para alcançar tal objetivo, é necessário trabalhar emsintonia, complementando suas ações, disatindo e alcançandb, sempre que possível, una condusão comum

O paciente assistido emuma UTl perde seu conta to direto comfaniliares e pessoas próximas, e é destituído, mesmo que temporariamente, da sociedade, de suas atividades e rotinas, tendo que se relacionar com desconhedidos e ficando exposto a situações 
constrangedoras, a umanbiente dferente e inóspito, deparando-se comoutros pacientes, por vezes em condições piores que a sua, além de outros fatores que acabampor gerar nedb e angústia e, conseqüentermente, podem provocar-lhe depressão que o expõe a uma maior fragilidade e debilitação de seu estado emocional. Nesse sentido, o padiente predisa ser respeitadb e atendidb emalguras de sues necessidades e direitos, como individualidade, privacidade, presença da fańlia e de profissionais que o acolhame o façam sentir o mais confortável possível, respeitando suas crenças, alturas e opiniões acerca de seu tratamento, e esdareçamsuas dúmidas.

A família é entendida, neste contexto, conro una unidade social proximamente conectada ao paciente através do anror, podendo ou não ter laços legais ou de consangüinidade. Segundo Santos et al.:28 :

A família deve ser compreendida como umaliado importante da equipe, podendo atuar como um rearso por micio do qual o paciente pode reafirmar e, mitas vezes, reauperar sua importância no tratamento, de forma a investir nas suas possibilidades de reauperação.

Quese sempre, a internação de umfamiliar tende a desestuturar a argarização dos papés capadbs por cada nentbro da famlia, posicionandb-os emuna situação de fraglicade dante do necb da perca do erte quericb

Unta intenvenção junto aos familiares é necessá ria, dando-lhes oportunidade para expor suas dúMdas, medos e sentimentos emrelação à internação do padiente na UTl, ajudandb-os a se conscientizarem da real situação do doente e da necessidade de tratamento na UT. É prediso garantir que as famílias sintamse apoiadas, úteis e participativas no tratanerto do padiente e terhamsuas dívidas esdaredidas.

Outro ponto fundamental no processo de humanização é entender a equipe de maneira interdisciplinar, atuando e potencializando as ações entre si, respeitando o potencial de cada um

Os profissionais de saúde que trabalhamemUTls são confrontados diariamente comquestões relativas à morte, às dificesis condições de trabalho (baixa remneração, trabalho excessivo, insuficiênaia de rearsos humanos e materiais, entre outros) que interferemdiretamente emseus sentimentos, emoções e defesas, podendo ser causas geradoras de estresse

O aidadb enrociond é de responsabalidade de toda a equipe de saúde, que precisa estar emcondições emocionais de trabalhar comos pacientes, seus familia res e comnidade Ser saudável é uma conquista que deve ser buscada não só para os pacientes, mas tam bémpara a vida dos profissionais que tuamemUlls.
Segundo Rodkenbadh:53, a desumanização da equipe, por falta de condições de trabalho, provoca a desumanização do padiente, que deixa de receber os aidados a que temdireito Boerer et $a l^{10}$ ainda dizemque aidar de quemaida é condição necessária para melhorar a qualidade do aidado na UT.

Nesse contexto, o trabalho teve por objetivo levantar na literatura cientúfica nacional dos útimos 10 anos artigos relevantes sobre humanização em unidades de terapia intensiva relacionada ao paciente adulto, família e equipe de enfermagem enfocando as principais estratégias utilizadas e dificuldades encontradas para sua implementação.

MÉTODO

Este estudb caracterizourse como sendo de natur reza descitiva, retrospectiva, de revisão bibliográfica sobre o tema humanização em unidades de terapia intensiva Utilizaramse como descritores de assunto os termos humanismo, unidades de terapia intensiva e enfermagem efetuando una busca nas bases de dados MEDNE, LLACS e SOBO Para escolha dos artigos definiramse como citérios de indusão, artigos nacionais publicados emperiódicos nos útitinos 10 anos que abordassem a temática proposta. Com os descritores humanismo, unidades de terapia intensiva e enfermagem obtiveramse 2 publicações indexadas ao LLACS e nenhuma no SalO $\mathrm{O}$ MDUNE Dante dessa pequena amostra obtida optourse pelo uso combinatório dos descritores de assuntos. Assim utilizaramse humanistro e unidades de terapia intensiva e foram encontradas 7 publicações indexadas ao MEDUNE e 14 ao LLACS. Já comos descritores humaristro e enfermagem, obtiveramase 11 publicações no MEDNE e 10 no LLACS. Por fim continaramse uridades de terapia intensiva e erfermagem, e foramdotidas 19 pudicações no MEDNEe 32 m LLACS Mestro coma combinação de descritores não se doteve nerhuma ocor-

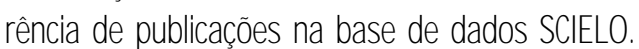

Do total de 95 publicações encontradas, apenes 15 atendiamaos critéios de indusão, sendo que delas, un publicação repetiurse no LLACS por 3 vezes, devido às combinações entre os descritores de assuntos; portanto, foi considerada apenas uma única vez. Dessa forma, a amostra do estudo foi de 12 artigos.

Conforme os artigos foramsendo obtidbs, recebe ramuna numeração de acordo como ano de publica ção, emordemcrescente, e foramanalisados de forma descritiva norteada pelos objeivos propostos. A delimitação dos artigos, bemcomo sua colocação em ordemcronológica, está representada no Quadro 1, 
que se encontra emanexo. A partir dos resultados e discussões apresentados a seguir, a numeração sobrescrita entre parêtteses referese aos artigos seledonados para a revisão.

RESULTADOS E DISCUSSÃO

Diante do levantamento bibliográfico realizado, buscourse identificar nas publicações selecionadas, através da leitura integral das mesmas, se os autores explicitavamemseus estudos estratégias utilizadas e dificuldades encontradas para a implementação da humanização em unidades de terapia intensiva, tanto relacionada aos pacientes como também às famílias e à equipe de enfermagem conforme a proposta do estudo.

Dificuldades da implementação da humanização para o paciente adulto e sua família e os profissionais de enfermagem em unidades de terapia intensiva

A análise dos textos selecionados pôde evidenciar que a maioria deles traz, emseu conteúdo, aspectos relacionados ao ambiente da UT que influenciam negativamente sobre a ação dos profissionais no aidado humarizado aos padientes e familiares, como, por exemplo, o apego à tecnologia ali empregada, sobrepondo a utilização rotineira dos equipamentos ao humano ali aidado e não percebido como umser emsua totalidade, que requer aiidados e direitos atendidos emsua plenitude(1, 5-8, 11, 12). Isso fica evidenciado na seguinte ditação de Ribeiro"1:16 :

É possível identificar que a tecnologia exerce umfascánio sobre os profissionais da saúde, todavia, ressalta-se, é imperioso atentar para que a máquina não se torne mais importante do que o próprio paciente.

Nascimento e Stipp ${ }^{12}$ acreditam que é de fundamental importância a atuação do enfermeiro gerencial de forma a evitar a sobreposição da tecnologia ao paciente cuidado, o que fica evidenciado a seguir:

De todos os aspectos ditados, o mais relevante de todbs é a forma como direcionar a assistênaia de enfermagem ou seja, transformar a prática gerencial numa dinâmica catalisadora de ações humanizadas e voltadas para o ser humano com necessidades espeáficas, evitando que a madça presença de equiparrentos e processos tecnológicos tornemo auidado de enfermagem una fábrica de montageme desmontagen 22:192.
Orrecanicistro e robotização das ações da equipe de enfermagem que por seremrotineiras e, mitas vezes ńgiclas e inflexiveis, tomamo aidadb ao padiente, impessoal, impositivo e fragmentado, dificultando, assim a prestação de umaidado humarizadb. 1,46,7,10,11

Villa e Rossi 7:141 afimam emseu estudo, que o objeto da enfermagemestá centrado mais na tarefa a ser exeatada do que no paderte.

As rodines de visita aos padiettes, que mites vezes rão tendamplenantente aos anseios e epectatives dos pacientes

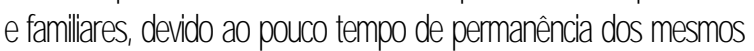
e à rigidez dos horários, gerandb ainda mais ansiedadee insegurança a antoos $3,6,9$.

Oiveira et al. ${ }^{13: 41}$ referemque os entrevistados expressaramseus sentimentos de pesar pela ausênia da fámilia (...) devido à rotina que restringe a entrada de pessoas no ambiente

Ainda, esse mesmo ambiente inóspito da UT pode gerar fatores estressantes tambémpara a equipe de enfermagem como os ditados abaix:

- As situações emocionalmente conflitantes e de estresse (risco iminente de vida, a constante presença da norte, o conúvio coma dor do autro) fazemparte do cotidiano da equipe prestadora de aidados na UT, podendo ailminar no desgaste físico e psicológico da equipe que, por vezes, adota mecarismos de defesa na tertativa de não sofrerem como sofrimento do autro, 3,5,7,9,10.

- Collet e Rozendbo'1919 afirmamque afeivamente a enfermagem pode assumir ura postura de indisponibilidade e de intolerância para coma emoção provocada pela percepşão do sofinimento do outro. Ao rejeitar ou negar o sofimento do usuário, a enfermagemse protege.

As instituições de saúde, onde o rodlo bionédico ainda é vigente, colaboram sobremaneira na dificuldade em implementar uma assistência humanizada aos seus pacientes, uma vez que não proporcionam à sua equipe condições dignas de trabalho, submetendo-a a tumos prolongados de trabalho, número insuficiente de pessoal, baixa remuneração, desvalorização das suas açães, que, comparadas às ações rédicas, são dassificadas com de menor importância, causando frustração e desmotivação emtoda a equipe, que, dessa forma, rãoé "aidada" ceforma hurarizada praque possa oferecer o mestro aidadb hunariadb aos padientes ${ }^{3,48,10}$.

Enseu estudb, Vlla e Rossi 7:141 afimamque

Nessa categoria, os informantes ressaltaram aspectos referentes ao estresse pela sobrecarga 
de trabalho e o intenso número de atividades no dia-a-dia da UT (...) Mendonaram ainda, a importância de estender a atenção à equipe de enfernagemno aidado humanizado, ou melhor, aidar de quemaida, como condição necessária para methorar a qualidade do aidado na UT.

Otras ditações apareceramnos demais artigos commenor freqüênda, o que não significa que sejam de menor importância, sendo que devem ser levadas emconsideração pelos profissionais e instituições na tentativa de humanizar o aidado prestado, a saber:

- Conflitos no relacionamento profissional-paciente e/ou profissional-famlia.

- O discurso em contradição às ações. Neste sertidb, Vlla e Rossi 7:141 relatamque

A dossenvação do contexto real cla terapia intensiva faz-nos constatar a dicotomia existente entre teoria e prática. Apesar de conceituarem aiidado humanizado como respeito, amor, carinho, mantendo o dálogo, a privacidade, dando atenção à família, os informantes mencionamatitudes, comportamentos, condutas que caracterizama UII como umanbiente recânico e desumano com paciente, familia e equipe de enfermagem

- Acompeitividadenos das atuais, tomandb profissionais einstituições egástes, indvid listes, concorrentes, em que o único objetivo é a produção de senviço, não importando como se dará essa prod ção? .

- Barilho constante dos equipanertos e conversas entre os profissionais, influenciando no descanso apropriado do paciente?

- Falta de privacidade referida pela maioria dos pacientes, expondb-os a situações constrangedoras que os fazemvivenciar a desapropriação de seu conpo, autonomia e liberdade de opinar'.

- Famílias que se sentemtotalmente exduídas e pouco participativas nos cuidados e tratamento de seu familiar, sentindo-se impotentes diante da situação de doença em que o paciente se encontra ${ }^{7,911}$

Dessa forma, esses foramos principais achados acerca das dificuldades encontradas para a implementação da humanização da assistêndia em Unidades de Terapia Intensiva.

Estratégias para a implementação da humanização ao paciente adulto, família e profissionais de enfermagem em unidades de terapia intensiva

Dentre os artigos que atendiamaos citérios de indusão preestabeledidos, poucos traziamsugestões ou nestro ações efetives compreendidas conø estratégias ra tertativa de humarizar a assistênja emterapia intensiva

Forammito citados, na visão de profissionais, pacientes e famílias, aspectos que tendema ir de encontro coma humanização do aidadb, mas que, no entanto, não encontramvias de seremaplicados na prática cotidiana, seja pela formação dos profissionais endvidos, seja pelo modb de trabalho emque se dá a assistêndia emunicades de terapia intensiva, ou nestro por se tratar, ainda, de uma ideologia, emque sua necessidade é compreendida por todbs, mas de difíil incorporação pessoal.

Agumes considerações seguemabaix:

- Oambiente ou estrutura física foi ditadb como de fundamental importânaia no bemestar de paciente, equipe e família, podendo constitur-se como um facilitador das ações na tentativa de humanizar a assistêndia, através da colocação de calendários, relógios (para situaremo paciente no tempo e espaço), jandas espalhadas, permitindo ao paciente ver o ambiente externo, dferenciar a luz do dia do anoitecer, facilitando percepções que são prejudicadas em um ambiente fechado ${ }^{7,3}$.

- A aplicação de una nova abordagemou modelo assistencial na tentativa da contribuição para o aidado de enfermagememterapia intensiva ${ }^{6,11}$. Nascimento e Trentini 15:251 propõema refleão sobre a possibilidade de o paradigma da simitaneidade, apoiado na teoria humanística de Paterson e Zderad, contribuir para a humarização do aidado. O presente estudo é apoiado emparticular, nas concepcõoes relatives ao ser humano, anbiente, saúde, enfermagem e diálogo, e em nossa experiência profissional, na área da terapia intensiva, segundo a ótica de Nascimento e Treitii ${ }^{15}$ e Martins e Farias ${ }^{16}$.

Enfim acreditamos que o paradigma da simitaneidade, como forma de perceber o ser humano hospitalizado emUII, possibilita umaidado que rompe como moddo assistencial predominante, ajo objeivo maior é a ara e não o aidadb do ser.

Ainda nesse contexto de una nova abordagemou modlo assistendial, Martins e Faria ${ }^{16398}$ consideramque Neste roddo [abordagemsócio-humarista], a equipe de enfermagem, rompe comsua forma tradicional de trabalho, emque o enfermeriro estipula as ações de aidadb e os trabalhadores de rível nédio (técicos e axiliares de enfermagem, auxiliar de saúde, assistente adhinistrativo) são simples exeatores desse aidadb. A equipe de enfermagem nesta proposta, se toma mediadora do processo de reaperação do sujeito hospitalizadb e os sujetos familiares. 
Os autores ditados consideramainda, que nesse modelo é permitida a realização de umtrabalho em que todos os envolvidos (paciente, família e equipe) contribuam ativamente; ou seja, a participação de todos é assegurada.

A comricação e o dálogo entre todos os envividos no processo de permanência de umpaciente na UTI foramitens citados emtrês textos ${ }^{5,8,11}$, comb ações que criamoporturidades às relações, otimizando as expressões, tanto djeitives quanto subjetives. Segundo Nascinento e Trentini15:257:

Oambiente da UT tomar-seá menos impessoal para o doente e seus familiares quando o diálogo estiver aberto para ambos, quando houver uma interação entre os doentes e seus familiares, entre eles e o ser aidador de enfermagem e entre os aidadores de enfermagemda UT.

Otras estratégias ditadas emumnúmero menor dentre os artigos foram

- Melhor envolvimento e interação efetiva com paciente e famlia’, 8 ,

- Necessidade de aidar de quemaida;

- Retorrar o valor afetivo do cuidado, o amor (enquanto momento de expressão do aidado), a empatia, a intimidade e a solidariedade no sentido de cuidar do outro como eu gostaria de ser aidado ${ }^{2,4}$.

\section{CONSIDERAÇÕES FINAIS}

Retomando o objetivo inicial desse trabalho, o de buscar na literatura nacional dos útimos 10 anos aspectos relevantes sobre as dificuldades e

\section{Referências}

1 MristéiocaSánde(BR). SerretaiadeAssistênjaàSáde Programa Neciond deH nmaizaçãodaAssistêndaHospatdar. Braslia(DF);2001

2. MristériodaSánde(BR). Manul dbH marizaSus, Braslia(DF). Dsporivel emuvurusa degov.br/humarizesus

3. SazaM PossaijF, MgaiarKłB. Huratzaçãodadbordagames unicades deterapaintensiva. PevPal Eferm1985abr, 5(2): 77-9.

4. Boff L Saberaidar: éticas dohumano; compaixãopelatera. Teed. Petrópolis(B)): Vozes; 1999. estratégias facilitadoras na implementação da assistêndia humanizada em Unidades de Terapia Intensiva relacionada a pacientes, família e equipe, pôde-se conduir que o tema "humanização" tem sido freqüente objeto de pesquisas dos profissionais da área da saúde e, emespedial, de enfermeiros, preocupados coma qualidade da assistência que temsido prestada aos seus padientes. No entanto, quando essas pesquisas propõe-se estudar e disatir o terma, elas se restringemmais a aspectos teóricos do que às reais possibilidades de sua implementação; ou seja, é mais no discurso do que na prática que essa discussão acontece.

Prendemse a conceitos e às opiniões de outros autores previamente publicadas e em virtude da realidade vivida dos pesquisadores, que, emquase sua totalidade, já experienciaramo ser enfermeiro em Unidades de Terapia Intensiva, eles relatamcommais facilidade as principais officuldades encontradas nesse setor para a implementação da humanização. Mas, quando da sugestão de estratégias viáveis para que isso aconteça, restringemse apenas a conduir o quão necessánia toma a reflexão e busca de ações fadilitadoras, semexplicitar como isso pode acontecer.

Então, conduímos que, enquanto os pesquisadores focaremseu objeto de estudo apenas no tema geral "humanização", não propondo ações e estratégias para que ela efetivamente aconteça, continuarão encontrando cada vez mais dificuldades para sua implementação. E a humanização da assistêndia emUTls continuará sendo uma ideologia distante da realidade.

5. CorreaAK Dotreinamerto do enfermero à possibilidadeda educaçãoenterapiaintensiva: embuscadb sentido daexistênia humana [Tese de Doutorado]. Riberião Preto (SP): Escola de EnfermagemdeRiberiãoPreto/ USP; 2000.

6. MezoñJA Oqueéhumarizaçãono hospita? Giralar 02/80 apresentadano 1ㄴongressoBrasileirodeH marizaçãodb Hospital eda Saúde, 1980; SãoPaulo(SP), Brasil.

7. VIllaVSC, ROSS LA Osignificadbattural do aidadbh manizadb emuricadedeterapiaintensiva "mitofaladbe parcovividb". Rev Latino-amEfremragem2002 mar/abr, 10(2): 137-44. 
8. Sartos RR ToledoNN SilvaSC Humanização emuridadede terapiaintensiva: padeiente equipedee efermagemfamlia. Nursing 1999 at; 17:26-9.

9. PodkerbachlH Aerfermagemeahumarizaçãodopadente Pev Bras Erferm1985, jar/mar, 38(1): 49-54.

10. Boemer MR Rossi LR Nestari RR Aidáademorteemuridade deterapia intensiva; análise de depoimentos. RevGaúchaEnferm 1989jul; 10(2):8-14.

11. RibeiroRCN CarandinaDM Fardh GGFugtaRM. Teanologiae humarização. RevSOBECC1999ju/set, 4(3):15-9.

12. NescimentoMIF, StippMAC Gerêndade Uhidadede Cuicado Gńtico. EscAmnaNkry RevErfermagem2002ago; 6(2): 189-94.

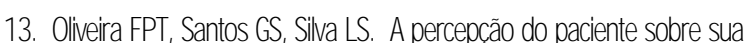

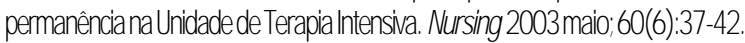

14. ColletN RozendbCA Humarizaçãoetrabalhonaenfermagem RevBrasEfferm2003mar/abr, 56(2): 189-192.

15. Nascimento $B P$, Trentini $M$ Oaidado de enfemagemna uricadedeterapiaintensiva(UT): teoriahumanísticadePatersone Zderad PevLdino-amEreremagem2004mar/abr; 12(2): 250-57.

16. Mertins JJ, Faria $E M A(r e)$ organização do trabalho da effermagememUII, através deu uma nova proposta assistencial. Texto\&ContetoEferm2000 mai/ago; 9(2): 388-401.

\section{Sobre as Autoras}

\section{Fabianbuda}

Espedalistaem EfermagemdeTerapialntensiva MestrandaemEnfermagem da Escola de Enfermagem de Ribeiro Preto - SP. fbolela@yohoo.combr

\section{MarlideGanalojericó}

Profedo Curso de EnfermagemdaFaculdadedeMkdicinade São Josédo RioPreto- SP. DoutorandaemEnfermagemdaEscolade ErfermagemdaUFP. marli@famerp.lor 


\section{ANEXO}

Qadro1: Artigosselecionedos queđendamaos citéín deindusãopreestabdecidbs, comseuscorrespondentes a tores, ano depublicação, periódicoebasededadbs ondeforamindexadbs.

\begin{tabular}{|c|c|c|c|}
\hline Referência & Estudo/Titule & Autce/Atio & Periodico'Base de Dades \\
\hline 1 & $\begin{array}{l}\text { Humanização em terapia } \\
\text { intensiva: a contribuiçăo da } \\
\text { abordagem fenomenológica }\end{array}$ & $\begin{array}{l}\text { SANTOS, L.C.G: } \\
\text { TOCANTINS, F.R } \\
1998\end{array}$ & $\begin{array}{l}\text { Rev. Enferm. UERI } \\
\text { LILACS }\end{array}$ \\
\hline 2 & $\begin{array}{l}\text { Cuidado solidário: um } \\
\text { compromisso social da } \\
\text { enfermagem em Unidades de } \\
\text { Terapia Intensiva }\end{array}$ & $\begin{array}{l}\text { BETTINELLI, L.A; } \\
\text { ERDMANN, AL. } \\
1988\end{array}$ & $\begin{array}{l}\text { Cogitare Enfermagem } \\
\text { UILACS }\end{array}$ \\
\hline 3 & $\begin{array}{l}\text { Humanizaçăo em Unidade de } \\
\text { Terapia Intensiva: paciente - } \\
\text { equipe de entermagem - familia }\end{array}$ & $\begin{array}{l}\text { SANTOS, C.R; TOLEDO, } \\
\text { N.N; SILVA, S.C } \\
1999\end{array}$ & $\begin{array}{l}\text { Nursing } \\
\text { LILACS }\end{array}$ \\
\hline 4 & $\begin{array}{l}\text { Humanizaçăo da prática pelo } \\
\text { cuidado: um marco de } \\
\text { referência para a enfermagen } \\
\text { em unidades criticas }\end{array}$ & $\begin{array}{l}\text { PAGANINI.M.C } \\
2000\end{array}$ & $\begin{array}{l}\text { Cogitare Enfermagem } \\
\text { ULACS }\end{array}$ \\
\hline 5 & $\begin{array}{l}\text { A humanizaçäo na UTi - um } \\
\text { caminho em construçăo }\end{array}$ & $\begin{array}{l}\text { SOUZA, L.NA_; PADILHA, } \\
\text { MIICS. } \\
2000\end{array}$ & $\begin{array}{l}\text { Texto Contexto } \\
\text { Enfermagem } \\
\text { LILACS }\end{array}$ \\
\hline 6 & $\begin{array}{l}\text { A (Re) Organização do trabalho } \\
\text { da enfermagem em UTL, através } \\
\text { de uma nova proposta } \\
\text { assistencial }\end{array}$ & $\begin{array}{l}\text { MARTINS, IL; FARIA, E.M. } \\
2000\end{array}$ & $\begin{array}{l}\text { Texto Contexto } \\
\text { Enfermagem } \\
\text { LILACS }\end{array}$ \\
\hline 7 & $\begin{array}{l}\text { O significado cutural do } \\
\text { cuidado humanizado em terapia } \\
\text { intensiva: "muto falado e pouco } \\
\text { vivido" }\end{array}$ & $\begin{array}{l}\text { VILLA, VS.C; ROSSI, LA, } \\
2002\end{array}$ & $\begin{array}{l}\text { Rev. Latino-americana } \\
\text { de Entermagem } \\
\text { ULACS }\end{array}$ \\
\hline 8 & $\begin{array}{l}\text { Humanizaçào e trabatho na } \\
\text { enfermagem }\end{array}$ & $\begin{array}{l}\text { COLLET, N; } \text { ROZENDO, } \\
\text { C.A. } \\
2003\end{array}$ & $\begin{array}{l}\text { Rev. Brasileira de } \\
\text { Enfermagem } \\
\text { MEDUNE }\end{array}$ \\
\hline 9 & $\begin{array}{l}\text { A percepção do paciente sobre } \\
\text { sua permanência na Unidade de } \\
\text { Terapia Intensiva }\end{array}$ & $\begin{array}{l}\text { OLIVEIRA, F.P.T: SANTOS, } \\
\text { 6.S.; SILVA, L.S. } \\
2003\end{array}$ & $\begin{array}{l}\text { Nursing } \\
\text { LILACS }\end{array}$ \\
\hline 10 & $\begin{array}{l}\text { Humanizaçāo da assisténcia de } \\
\text { enfermagem estudo com } \\
\text { clientes no periodo pós- } \\
\text { interraçáo de uma UTI-adulto }\end{array}$ & $\begin{array}{l}\text { MATSUDA, L.M.; SILVA, N.; } \\
\text { TISOLIN, A.M. } \\
2003\end{array}$ & $\begin{array}{l}\text { Acta sci, Health sci } \\
\text { ULACS }\end{array}$ \\
\hline 11 & $\begin{array}{l}\text { O cuidado de enfermagem na } \\
\text { Unidade de Terapia Intensiva } \\
\text { (UTI): teoria humanistica de } \\
\text { Paterson e Zderad. }\end{array}$ & $\begin{array}{l}\text { NASCIMENTO, E.R.P.; } \\
\text { TRENTIN, } M . \\
2004\end{array}$ & $\begin{array}{l}\text { Rev. Latino-americana } \\
\text { de Enfermagem } \\
\text { MEDUNE }\end{array}$ \\
\hline 12 & $\begin{array}{l}\text { Significado do cuidar na } \\
\text { Unidade de Terapia Intensiva }\end{array}$ & $\begin{array}{l}\text { LUCENA, A.F; CROSSETI, } \\
\text { M.G. } \\
\text { ZOO4 }\end{array}$ & $\begin{array}{l}\text { Rev. Gaúcha de } \\
\text { Enfermagen } \\
\text { MEDLINE }\end{array}$ \\
\hline
\end{tabular}

